\title{
28 Research Square \\ The significance of blood cell biomarkers in the prognosis of gastric cancer
}

\author{
Pu Huang \\ Bejing Cancer Hospital \\ Yiran Zhang \\ Beijing Cancer Hospital \\ Anqiang Wang \\ Beijing Cancer Hospital \\ Zhao-de Bu ( $\nabla$ buzhaodebjc@163.com )
}

Research article

Keywords: Gastric cancer, Biomarker, prognosis, inflammation

Posted Date: June 23rd, 2020

DOI: https://doi.org/10.21203/rs.3.rs-33702/v1

License: (c) (i) This work is licensed under a Creative Commons Attribution 4.0 International License.

Read Full License 


\section{Abstract}

Background Studies have shown that inflammation-associated blood cell markers are associated with prognoses in a variety of tumors. However, the prognostic significance of these markers for gastric cancer (GC) is still not very clear. This article aims to explore its value of GC prognostic assessment.

Methods From July 2011 to July 2016, 353 GC patients with surgical treatment were enrolled in this retrospective study. Patients' demographics were analyzed along with clinical and pathologic data. The chi-square test was used to evaluate relationships between the markers and other clinicopathological variables; The Kaplan-Meier method and Cox regression proportional hazard model were performed to evaluate prognostic factors.

Results Univariate analysis indicated T stage, N stage, vascular tumor thrombus, tumor long diameter, Bormann Classification, preoperative MWR (monocyte/leukocyte ratio), preoperative serum CEA levels are prognostic factors for GC. Multivariate analysis showed that preoperative MWR, tumor differentiation, and tumor length were independent prognostic factors in patients with GC. The boundary value of MWR is 0.8 .

Conclusion Preoperative MWR was convenient, simple marker of gastric cancer, might be useful for the evaluation of prognosis of patients with GC. Comparing with TNM stage, tumor differentiation was a more reliable pathological factor evaluating recurrence.

\section{Background}

Gastric cancer (GC) is one of the most common tumors worldwide. In 2015,the number of deaths caused by gastric cancer in China ranked second among all tumor-related deaths[1]. Most patients with gastric cancer are already in advanced or even advanced stage of diagnosis because it lacks early specific symptoms and signs, and it often progresses rapidly. Although there are many treatments for gastric cancer, surgical resection is still the only way to cure patients. Postoperative metastasis and recurrence are the most common causes of death in patients with gastric cancer[2,3]. It is currently believed that preoperative status, treatment plan, postoperative TNM stage, pathological features of the tumor (Bormann classification, Lauren type, degree of differentiation) and other factors can affect the prognosis of gastric cancer[3]. However, these markers can only be obtained after surgery. Therefore, we need some new markers which able to predict the risk of recurrence in patients with gastric cancer.

In recent years, studies have shown that systemic inflammatory response is closely related to tumor progression and invasion. In addition, there is a large amount of evidence that the inflammatory response is earlier than the malignant transformation of tissue cells, which promotes the development of tumors [4]. Nowadays, many studies have reported that some hematological markers and scoring systems can simplify and standardize the measurement of systemic inflammatory response to clinical practice. Including C-reactive protein ( CRP) / albumin (ALB ), neutrophil / lymphocyte ratio (NLR), platelet / lymphocyte ratio (PLR), etc[1, 2, 4-8]. Besides, blood test markers: neutrophil-to-1ymphocyte ratio (NLR), 
platelet to lymphocyte ratio (PLR), monocyte to lymphocyte ratio (monocyte to lymphocyte ratio, MLR) and coagulation test markers: fibrinogen (Fbg), can reflect the balance between tumor inflammatory levels and anti-tumor immunity[9-11]. In addition, there are many combinations of inflammatory cell markers such as lymphocyte-to-white blood cell ratio (LWR), neutrophil-to-white blood cell ratio (NWR), monocytes. /Monocyte-to-white blood cell ratio (MWR) also has a certain significance in tumor prognosis[12, 13].

Studies have shown that more than $15 \%$ of tumor pathological sections around the inflammatory cell infiltration can be observed through histopathological examination [14] and the inflammatory response is a non-specific reaction in the body that may be associated with ischemia, hypoxia, tissue damage, and tumor necrosis in tumor progression[12]. During the progression of the tumor, local inflammatory reactions lead to changes in the neuroendocrine system and tissue blood supply, which is the main mechanism for tumor cells to escape the immune system of the body and continue to develop $[15,16]$. Although studies have confirmed the relationship between some inflammatory markers and gastric cancer, but others like LWR, NWR, and MWR are still unclear. Therefore, further research is needed to clarify the relationship between gastric cancer and inflammatory markers, especially LWR, NWR, MWR $[17,18]$.

\section{Methods}

\section{Patients}

A total of 353 patients who underwent gastric cancer surgery (radical surgery + palliative surgery + abdominal exploration) from July 2011 to July 2016 at Peking University Cancer Hospital were collected. The clinical data were obtained from the medical records of the patients in the hospital.

\section{Case selection}

(1) Patients who have been diagnosed with gastric cancer by endoscopic pathology or postoperative pathology at Peking University Cancer Hospital; (2) No blood transfusion, chemotherapy, infection, etc. or other related factors may affect blood routine and coagulation function before data collection Abnormalities; (3) recurrence of gastric cancer within 3 or 3 years of follow-up; (4) patient history, family status, physical examination report and other complete information, and no other tumors.

\section{Exclusion criteria}

(1) perioperative death; (2) death due to non-neoplastic disease; (3) combined with other tumors; (4) preoperative detection of acute inflammation or rheumatoid arthritis and other autoimmune diseases (5) The results of routine blood tests were absent within one week before surgery; (7) acute serious complications or death occurred within 30 days after surgery. (8) No radical surgery or palliative resection. (9) Patients receiving neoadjuvant chemotherapy (10) missing complete pathology report after operation 
After screening according to inclusion criteria and exclusion criteria, a total of 353 patients were included in the retrospective study. These patients had complete clinical medical records, pathology data, and follow-up data. The above study was approved by the Ethics Committee of Peking University Cancer Hospital.

\section{Data collection}

The patient's basic condition, laboratory test results, preoperative colonoscopy and pathology report, postoperative routine pathology report, and surgical pathological stage were collected. The basic conditions include age, height, weight, and surgical procedure; laboratory tests are complete preoperative blood routine results, including white blood cell count, neutrophil count, lymphocyte count, monocyte count, platelet count, platelet distribution width, Average platelet volume, hemoglobin, red blood cell distribution width, hematocrit. The above data were obtained through the medical record retrieval system. All patients signed informed consent before surgery, and the specific surgical procedures were performed according to relevant guidelines and specifications. The surgical approach can be divided into two types: radical and non-radical surgery. Radical surgery (R0 resection) refers to complete resection of the tumor, lymph node dissection, and negative margin. Non-radical surgery means that residual tumor tissue can still be seen under the microscope (R1 resection) or the naked eye (R2 resection) with the tumor removed and the lymphatic tissue removed. The margin is positive. The pathological type, TNM stage, Bormann type and degree of differentiation of the tumor were determined by postoperative pathology, and the eighth stage of AJCC TNM stage was used for tumor stage.

\section{Follow-up}

All patients underwent a return visit. The follow-up data was obtained by outpatient medical records, telephone follow-up or personal email. The contents include regular physical examination, imaging examination, laboratory examination and so on. The study endpoint was set to disease-free survival, defined as the time between surgery dates from imaging or pathological evidence of tumor recurrence. Recurrence was defined as a new lesion identified by imaging or histopathology during follow-up. The follow-up deadline was 2018-1-17.

\section{Factor definition}

NLR = neutrophil count / lymphocyte count; PLR = platelet count / lymphocyte count; MLR = monocyte count / lymphocyte count; NWR = neutrophil count / white blood cell count; LWR = lymphocyte count / white blood cell Count; MWR = monocyte count $/$ white blood cell count.

\section{Statistical methods}

The best cut-off point (cutoff value) of each factor is determined according to the ROC (receiver operating characteristic curve). For patients with an area under the ROC curve of less than 0.5 , the index with the reference range takes the upper limit level, and the factor with no reference range takes the median or mean according to whether the data conforms to the normal distribution. The continuous variables are 
expressed as (mean + standard deviation) and the Kolmogorov-Smirnov test is used to test whether the sample data conforms to a normal distribution. Nominal variables between the two groups were compared using a chi-square test or Fisher's exact probability test, and the ordered variables between the two groups were compared using the Wilcoxon test, and continuous variables between the two groups were compared using the $t$ test (bilateral significance level of approximately 0.05 ). The Kaplan-Meier method was used to estimate the disease-free survival time of the tumor, and the survival curve was drawn. If the important prognostic factors were basically balanced, the survival curves of the two groups were compared by Log-rank test. If important prognostic factors are not balanced, the Cox proportional hazards model is used to adjust and compare the survival curves of the two groups. The results of the analysis were generated by the spss 20.0 software. All the statistics were tested by two-sided test. When the $P$ value was less than or equal to 0.05 , the difference was considered statistically significant.

\section{Results}

\section{GC patient baseline data}

The clinicopathological features of patients with GC are shown in Table 1. A total of 356 patients with complete follow-up data were included in the study. There were 240 males (67.4\%) and 113 females (32.6\%). The average age of the patients was 58 years (23-84 years). Ten patients lacked postoperative T stage data; 8 patients lacked postoperative $\mathrm{N}$ stage data; 2 patients lacked Bormann type data; 3 lacked vascular tumor thrombus data; 223 lacked neurological invasion data; 72 lacked tumors Long-distance data; 46 people lacked Lauren type; 59 people lack Her-2; 59 people lack Ki-67; 4 people lack white ball ratio(Table 1).

\section{Threshold values of each factor}

Each factor was calculated based on the preoperative peripheral blood test results of the patient (Table 2). Evaluate whether the data is in a normal distribution and determine the critical value.

\section{Survival analysis}

Univariate analysis showed that T stage $(p<0.001)$ (Figure 1), N stage $(p<0.001)$ (Figure 2), Vascular tumor thrombus $(p<0.001)$ (Figure 3$)$, maximum tumor length $(p=0.047)$ (Figure 4$)$, Bormann classification ( $p=0.047)$ (Figure 5), M stage $(p=0.013)$ (Figure 6), MWR $(p<0.001)$ (Figure 7), and CEA levels ( $p=0.017$ ) (Figure 8 ) were all prognostic factors of GC; while Ki-67, Her-2 level, Neurological invasion, Lauren type, age, gender, BMl, degree of differentiation, PLR, MLR, NWR, LWR, NLR, Alb, white ball ratio, fibrinogen, hemoglobin are not factors influencing the prognosis of gastric cancer. Next, Cox multivariate analysis was performed on the statistically significant results and the influencing factors that were clinically associated with prognosis (Table 3). The results showed that preoperative MWR, maximum tumor length and tumor differentiation were independent risk factors for the prognosis of gastric cancer. 
The clinicopathological features between high and low group of MWR, NLR, MLR, LWR, NWR, PLR, Fbg were analyzed and summarized in Tables 4-10. Respectively, the distribution of clinical data and pathological factors between the two subgroups of MWR is shown in Table 4. Significant difference was observed in postoperative pathological T stage $(p=0.019)$ and hemoglobin levels $(p=0.02)$. However, no significant difference was found in other of the factors between the two subgroups. Based on the cutoff value, patients were classified in the two subgroups of NLR in Table 5, significant differences were observed according to tumor length $(p=0.047)$, ki-67 $(p=0.021)$ and preoperative CEA levels $(p=0.01)$. In the two subgroups of MLR, a statistically difference was found in age $(p=0.021)$, tumor length and preoperative hemoglobin levels (Table 6). In the two subgroups of LWR, a statistically difference was found in tumor long diameter $(p=0.05)$, Ki-67 level $(p=0.039)$, preoperative hemoglobin level $(p=0.049)$, preoperative CEA $(p<0.01)$ (Table 7$)$.In the two subgroups of NWR, a statistically significant difference in preoperative CEA levels was observed between the two groups $(p=0.035)$, while other factors did not (Table 8).In the two subgroups of PLR, significant differences were observed according to T stage ( $p=$ 0.024), preoperative Hb levels $(p<0.01)$, preoperative CEA levels $(p<0.01)$, the Her-2 level $(p<0.01)$ and tumor long diameters $(p<0.01)$ (Table 9$)$. In the two subgroups of Fbg, Significant differences were found in $\mathrm{N}$-stage $(p=0.01)$, a vascular tumor thrombus $(p=0.033)$, and in Lauren type $(p=0.03)($ Table10).

\section{Discussion}

As early as 1863, Virchow put forward the argument that the body's inflammatory response is related to tumor development, invasion, and metastasis [19]. Since then, a large number of studies have shown that systemic inflammatory response is not only involved in the occurrence and development of tumors, but also in association with tumor invasion and metastasis in vivo. Although the relationship between tumor biological behavior and systemic inflammatory response is still uncertain, but the relationship between them seems to be mutual. On the one side, the development of tumors can promote the infiltration of related inflammatory cells, on the another side the infiltration of corresponding inflammatory cells is accompanied by the production of inflammatory mediators, aggravating the inflammatory response in the body[20,21]. Also, tumor angiogenesis may be associated with inflammatory cells or inflammatory mediators, including IL-6, TNF, neutrophils, and so on. Moreover, these inflammatory cells or inflammatory mediators can also penetrate into the microenvironment of the tumor, promoting tumorigenesis, invasion and metastasis, thereby affecting the prognosis of cancer patients[22-25]. In terms of gastric cancer, NLR, PLR, Fbg, and MLR have been shown to have a certain correlation with its occurrence and development. Bur the relationship between NWR, MWR, LWR and gastric caner is still not certain. This research system reviews the common inflammatory markers as follows:

(1) NLR, PLR and MLR

Neutrophils are the most common type of leukocyte, and the ratio of neutrophils to white blood cells is a commonly used factor for judging the level of inflammatory response in the clinic. Recent studies have shown that hematopoietic factors secreted by tumors can up-regulate neutrophil levels in peripheral blood, and neutrophil levels in invasive tumor patients are higher than those in invasive patients[26]. In 
addition, studies have also reported that neutrophils can not only stimulate tumor growth by inhibiting the production of tumor necrosis factor, but also secrete substances including matrix metalloproteinase-9, vascular endothelial growth factor and related cytokines to promote tumor progression[27-29]. NLR is defined as the ratio of neutrophils to lymphocytes in peripheral blood. There is increasing evidence that NLR can reflect the balance between tumor inflammatory response and anti-tumor immunity. In addition, NLR has also been shown to have a certain relationship to the prognosis of tumors [30-33].

In this study, it suggests that NLR, PLR, and MLR are not independent risk factors for the prognosis of patients with GC through multivariate analysis, which is inconsistent with the results of some gastric cancer studies. In the univariate analysis, we found that NLR was not associated with disease-free survival, but PLR and MLR had some effects. It can be observed from the two survival curves that these two markers have a tendency to separate from disease-free survival, but have not reached statistical difference. The reason for this may be as follows: firstly, this study takes 3 years as the end point of the study, and the time is relatively short, which may not be enough to reflect the patient's true outcome. Secondly, there are more biases in the retrospective analysis, or some factors of the study, such as radiotherapy and radical surgery, require more sample data to verify.

\section{(2) MWR, LWR, NWR}

At present, there are few studies on these three types of blood inflammatory markers. This study found that patients' MWR levels are related to the disease-free survival rate of patients. Multivariate analysis found that MWR is an independent risk factor for the prognosis of patients with gastric cancer. In theory, the level of white blood cells is positively correlated with the degree of inflammatory response in the body, except in patients with severely infected myelosuppression. However, the results of this study suggest that: elevated MWR often suggests a poor prognosis for patients, that is, monocytes are positively correlated with patient prognosis, and white blood cells are negatively correlated with patient outcomes. The possible causes are as follows: 1 . As the tumor progresses, the rise of monocytes and leukocytes is not parallel, the monocyte rises much faster than the white blood cells; 2 . In patients with a higher degree of tumor progression, the white blood cell level is lower. Normal level; 3 , the level of white blood cells has nothing to do with the survival of patients, only the level of monocytes affects the prognosis of patients. Multivariate analysis indicated that MMW levels, tumor long diameter, and tumor differentiation were independent risk factors for gastric cancer patients. Classic TNM stage is not an independent risk factor, which is inconsistent with clinical experience. The reason may be as follows: 1 . The sample size of this experiment is small, and the follow-up data time is less than 5 years. 2. This study used DFS (disease-free survival time) to judge the prognosis of patients, while the traditional study of TNM stage and OS (Overall Survival) is more, and its relationship with DFS is worth further exploration.

(3) Fbg (fibrinogen)

Plasma fibrinogen ( $\mathrm{Fbg}$ ) is synthesized and secreted by liver cells. As a glycoprotein, plasma fibrinogen is directly involved in the blood coagulation process in the form of a blood coagulation factor. Clinically, 
Fbg is a sensitive factor of the reaction of fibrinolytic system and hypercoagulable state. Not only that, fibrinogen is also considered to be an adhesion factor involved in tumor cell proliferation, angiogenesis and metastasis[34, 35]. A large number of studies have shown that high levels of fasting igg in the body suggest a poor prognosis in patients with various cancers including nasopharyngeal cancer, esophageal cancer, colorectal cancer, and prostate cancer[29-31]. The negative results of this study may be related to the small sample size and short observation time of this study, suggesting that further research is needed.

\section{Conclusion}

The detection of blood inflammatory markers is convenient, simple and useful. However, in blood inflammatory markers, only MWR is an independent risk factor for predicting the prognosis of patients with gastric cancer and is expected to be used to guide patients with further treatment after GC. Besides, the pathological characteristics of the tumor itself (degree of differentiation and tumor diameter) can better predict the risk of recurrence in gastric cancer patients.

\section{Abbreviations}

GC, gastric cancer; NLR, neutrophil-to-lymphocyte ratio; PLR, platelet-to-lymphocyte ratio; ALT, alanine aminotransferase; MLR, monocyte-to-lymphocyte ratio; NWR, neutrophil-to-white blood cell ratio; LWR, lymphocyte-to-white blood cell ratio; MWR, monocyte-to-white blood cell ratio; Fbg, fibrinogen; TNM, tumor node metastasis; ROC, receiver operator characteristic; Cl, confidence interval; IL-6, interleukin-6; TNF, tumor necrosis factor; CEA, carcinoembryonic antigen; CA19-9, carbohydrate antigen 19-9; OS, overall survival; RR, relative risk; HR, hazard ratio; DFS, Disease-free survival.

\section{Declarations}

\section{Ethics approval and consent to participate}

This study was performed in accordance with the Declaration of Helsinki and was approved by the Ethics Committee of Peking University Cancer Hospital (Reference No. 2006021). Informed consent was obtained from each patient.

\section{Consent for publication}

Not applicable.

\section{Availability of data and materials}

The datasets generated and analyzed during the current study are not publicly available due to IRB regulation but are available from the corresponding author on reasonable request.

\section{Competing interests}


The authors declared that they have no competing interests.

\section{Funding}

Not applicable.

\section{Authors' contributions}

Pu Huang wrote the manuscript with support from Yiran Zhang; Anqiang Wang designed the study, Yiran Zhang performed the statistical analysis. Zhaode Bu supervised the project. All authors collected and interpreted the data, reviewed the manuscript. All authors read and approved the final manuscript.

\section{Acknowledgements}

We thank the staff members at the database center at Peking University Cancer Hospital and Institute for assistance with the data search and project management.

\section{Author details}

1Department of Gastrointestinal Surgery of Peking University Cancer Hospital and Institute: Performing standard radical gastrointestinal surgery and multidisciplinary treatment, learning center of multidisciplinary treatment supported by Chinese Anticancer Association and The Chinese Medical Association, IASGO CME Center.

\section{References}

1. Chen W, Zheng R, Baade PD, Zhang S, Zeng H, Bray F, Jemal A, Yu XQ, He J. Cancer statistics in China, 2015. Ca Cancer J Clin. 2016;66(2):115-32.

2. Waddell T, Verheij M, Allum W, Cunningham D, Cervantes A, Arnold D. Gastric cancer: ESMO-ESSOESTRO Clinical Practice Guidelines for diagnosis, treatment and follow-up. Annals of Oncology.

3. Tomasello G, Ghidini M, Liguigli W, Ratti M, Toppo L, Passalacqua R. Targeted therapies in gastric cancer treatment: where we are and where we are going. Invest New Drugs. 2016;34(3):1-16.

4. Feng F, Tian Y, Liu S, Zheng G, Liu Z, Xu G, Guo M, Lian X, Fan D, Zhang H. Combination of PLR, MLR, MWR, and Tumor Size Could Significantly Increase the Prognostic Value for Gastrointestinal Stromal Tumors. Medicine. 2016;95(14):e3248.

5. Je Y, Giovannucci E. Coffee consumption and risk of endometrial cancer: findings from a large up-todate meta-analysis. Int J Cancer. 2012;131(7):1700-10.

6. Wenjie J, Yili W, Xiubo J. Coffee and caffeine intake and breast cancer risk: an updated doseresponse meta-analysis of 37 published studies. Gynecol Oncol. 2013;129(3):620-9.

7. Green C, De Dauwe P, Boyle T, Tabatabaei S, Fritschi L, Heyworth J. Tea, Coffee, and Milk Consumption and Colorectal Cancer Risk. Journal of Epidemiology. 2014;24(2):146. 
8. Feiyue X, Dan W, Zhifang H, Yajun G. Coffee consumption and risk of gastric cancer: a large updated meta-analysis of prospective studies. Nutrients. 2014;6(9):3734-46.

9. Rong Y, Chen L, Zhu T, Song Y, Yu M, Shan Z, Sands A, Hu FB, Liu L. Egg consumption and risk of coronary heart disease and stroke: dose-response meta-analysis of prospective cohort studies. BMJ. 2013;346(5):e8539.

10. Hildebrand JS, Patel AV, Mccullough ML, Gaudet MM, Chen AY, Hayes RB, Gapstur SM. Coffee, tea, and fatal oral/pharyngeal cancer in a large prospective US cohort. Am J Epidemiol. 2013;177(1):508.

11. Raul ZR, Leila LB, Bas H, Dik BDM, Heiner VK, Annika B, Anne S, Anja TN, Bodil Hammer O, Kim B. O: Tea and coffee consumption and risk of esophageal cancer: the European prospective investigation into cancer and nutrition study. Int J Cancer. 2014;135(6):1470-9.

12. Dominianni C,., Berndt W-YH,. S, Hayes RB, Ahn J. Prospective study of the relationship between coffee and tea with colorectal cancer risk: the PLCO Cancer Screening Trial. Br J Cancer. 2013;109(5):1352-9.

13. Dik VK, Bueno-De-Mesquita HBA, Oijen MGH, Van, Siersema PD, Uiterwaal CSPM, Gils CH, Van, Duijnhoven FNJB, Van, Stéphane C, Loic Y, Philippe F. Coffee and tea consumption, genotype-based CYP1A2 and NAT2 activity and colorectal cancer risk-results from the EPIC cohort study. Int J Cancer. 2014;135(2):401-12.

14. Kubota T, Hiki N, Nunobe S, Kumagai K, Aikou S, Watanabe R, Sano T, Yamaguchi T. Significance of the Inflammation-Based Glasgow Prognostic Score for Short- and Long-Term Outcomes After Curative Resection of Gastric Cancer. J Gastrointest Surg. 2012;16(11):2037-44.

15. Jiang N, Deng JY, Liu Y, Ke B, Liu HG, Liang H. The role of preoperative neutrophil-lymphocyte and platelet-lymphocyte ratio in patients after radical resection for gastric cancer. Biomarkers. 2014;19(6):444-51.

16. Nakayama Y, Gotohda N, Shibasaki H, Nomura S, Kinoshita T, Hayashi R. Usefulness of the neutrophil/lymphocyte ratio measured preoperatively as a predictor of peritoneal metastasis in patients with advanced gastric cancer. Surg Today. 2014;44(11):2146-52.

17. Jin H, Zhang G, Liu X, Liu X, Chen C, Yu H, Huang X, Zhang Q, Yu J: Blood neutrophil-lymphocyte ratio predicts survival for stages III-IV gastric cancer treated with neoadjuvant chemotherapy. World Journal of Surgical Oncology,11,1(2013-05-24) 2013, 11(1):112-112.

18. Qi J, Chang Y, Li B, Li X, Li X, Lv L. Clinical significance of preoperative inflammatory parameters in gastric cancer patients. Journal of Modern Oncology. 2013;13(2):111-6.

19. Balkwill F, Mantovani A: Inflammation and cancer. back to Virchow? Lancet 2001, 357(9255):539545.

20. Argilés JM, López-Soriano FJ. The role of cytokines in cancer cachexia. Med Res Rev. 2015;19(3):223-48.

21. Roxburgh CS, Mcmillan DC. Role of systemic inflammatory response in predicting survival in patients with primary operable cancer. Future Oncol. 2010;6(1):149-63. 
22. Katriina $H$, Shah E, Lawlor DA. A systematic review of the association between circulating concentrations of $C$ reactive protein and cancer. J Epidemiol Community Health. 2007;61(9):824-32.

23. Kusumanto YH, Dam WA, Hospers GAP, Meijer C, Mulder NH. Platelets and Granulocytes, in Particular the Neutrophils, Form Important Compartments for Circulating Vascular Endothelial Growth Factor. Angiogenesis. 2003;6(4):283-7.

24. Mary P, Rowley DA, Hans S. Inflammation as a tumor promoter in cancer induction. Semin Cancer Biol. 2004;14(6):433-9.

25. Ulich TR, Castillo J, Del, Guo KZ. In vivo hematologic effects of recombinant interleukin-6 on hematopoiesis and circulating numbers of RBCs and WBCs. Blood. 1989;73(1):108-10.

26. Lian L, You-You X, Chong Z, Xiao-Ming S, Xiang-Li L, Shu-Guang H, Yan Z, Zhong-Qi M, Fei-Ran G, Meng-Yao W. Application of platelet/lymphocyte and neutrophil/lymphocyte ratios in early diagnosis and prognostic prediction in patients with resectable gastric cancer. Cancer Biomarkers. 2015;15(6):899-907.

27. Yoshio O, Jun N, Makoto O, Tadashi H, Masaaki T. Pretreatment neutrophil-to-lymphocyte ratio as an independent predictor of recurrence in patients with nonmetastatic renal cell carcinoma. J Urol. 2010;184(3):873-8.

28. Bausch D, Pausch T, Krauss T, Hopt UT, Fernandez-Del-Castillo C, Warshaw AL, Thayer SP, Keck T. Neutrophil granulocyte derived MMP-9 is a VEGF independent functional component of the angiogenic switch in pancreatic ductal adenocarcinoma. Angiogenesis. 2011;14(3):235.

29. Kar Wai T, Zhen CS, Wong FHS, Maximilien E, Sandra Min-Li T, Jo K, Kemeny DM, Guan NL, JeanPierre A, Véronique A. Neutrophils contribute to inflammatory lymphangiogenesis by increasing VEGF-A bioavailability and secreting VEGF-D. Blood. 2013;122(22):3666-77.

30. Chen J, Hong D, Zhai Y, Shen P. Meta-analysis of associations between neutrophil-to-lymphocyte ratio and prognosis of gastric cancer. World Journal of Surgical Oncology. 2015;13(1):122.

31. Ethier JL, Desautels D, Templeton A, Shah PS, Amir E. Prognostic role of neutrophil-to-lymphocyte ratio in breast cancer: A systematic review and meta-analysis. Breast Cancer Res. 2017;19(1):2.

32. Marchioni M, Primiceri G, Ingrosso M, Filograna R, Castellan P, Francesco PD, Schips L. The Clinical Use of the Neutrophil to Lymphocyte Ratio (NLR) in Urothelial Cancer: ASystematic Review. Clin Genitourin Cancer. 2016;14(6):1558767316300982.

33. Yang $X$, Huang Y, Feng JF, Liu JS: Prognostic significance of neutrophil-to- lymphocyte ratio in esophageal cancer. a meta-analysis. Oncotargets \& Therapy 2015, 2015(default):789.

34. Wang Y, Yin W, Wang Z, Huang J, Pan J, Zhu Y, Xu F, Shao X, Sha J, Cai Y. Pretreatment plasma fibrinogen as an independent prognostic indicator of prostate cancer patients treated with androgen deprivation therapy. Prostate Cancer Prostatic Dis. 2016;19(2):209-15.

35. Fu SJ, Ji F, Han M, Chen MG, Wang XP, Ju WQ, Zhao Q, Wu LW, Ren QQ, Guo ZY. Prognostic value of combined preoperative fibrinogen and neutrophil-lymphocyte ratio in patients with hepatocellular carcinoma after liver transplantation. Oncotarget. 2017;8(3):4301. 


\section{Tables}

Due to technical limitations, tables are only available as a download in the supplemental files section

Figures

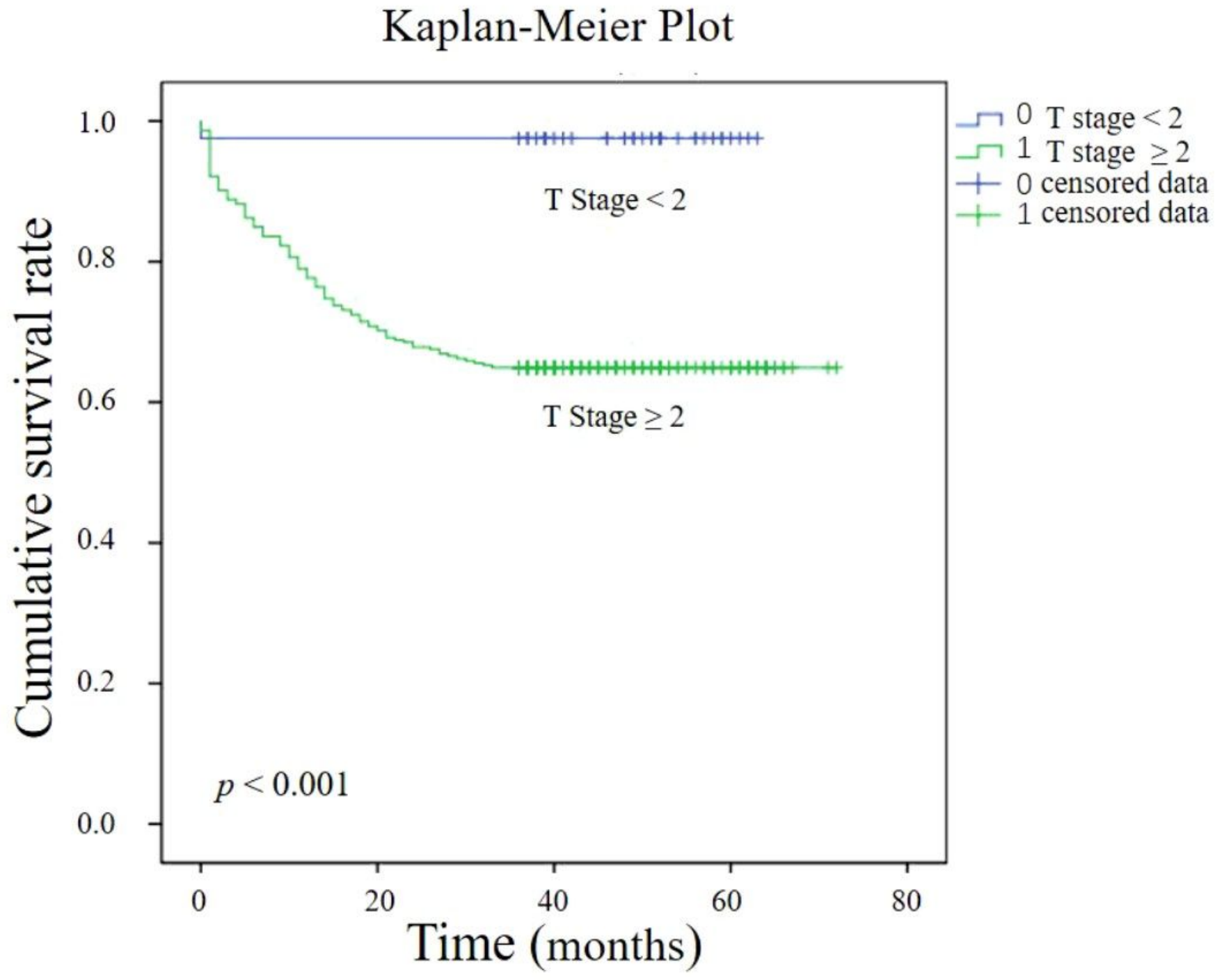

Figure 1

The Kaplan-Meier survival curves of DFS for patients stratified according to prognostic factors: T stage 


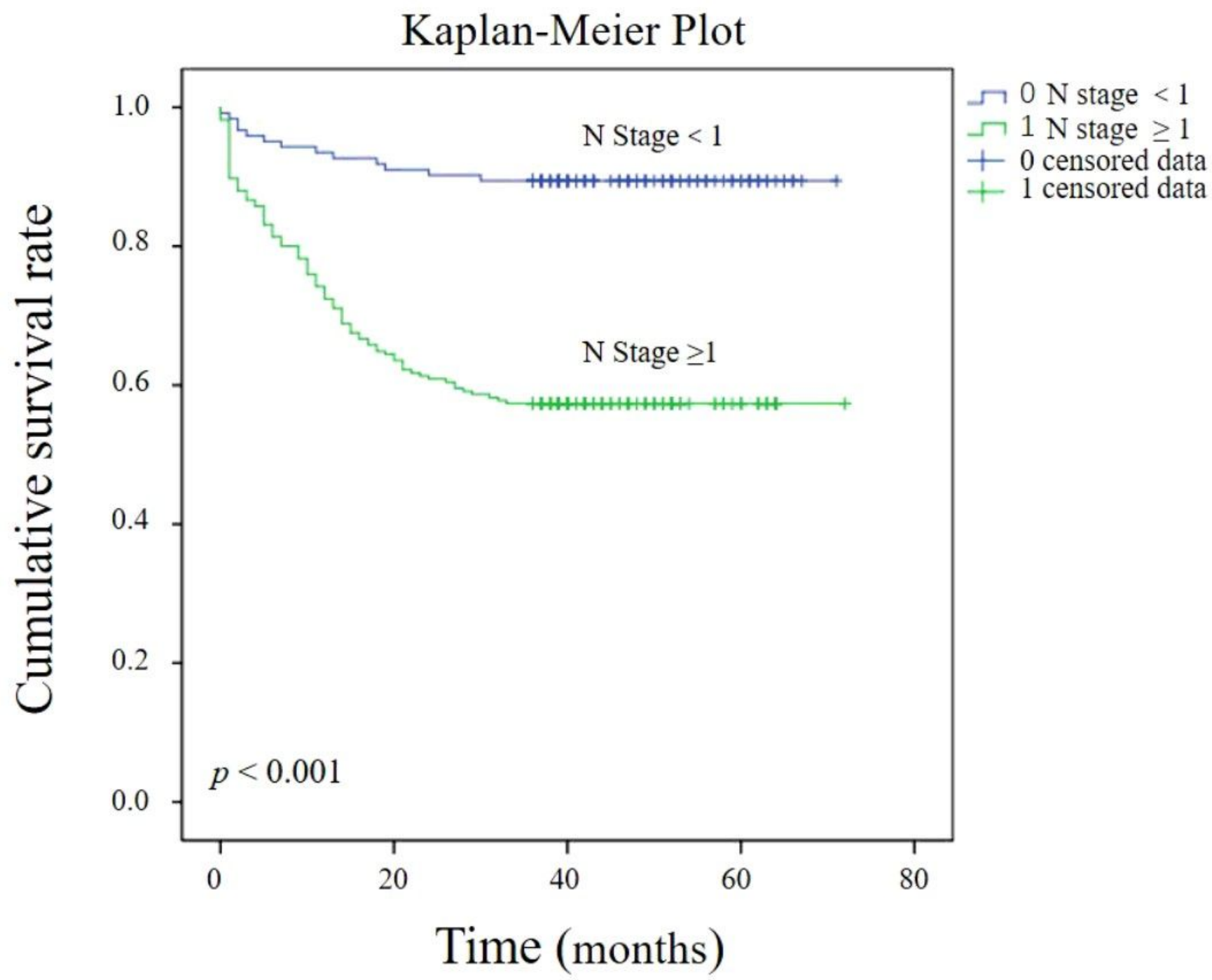

Figure 2

The Kaplan-Meier survival curves of DFS for patients stratified according to prognostic factors: $\mathrm{N}$ stage 
Kaplan-Meier Plot

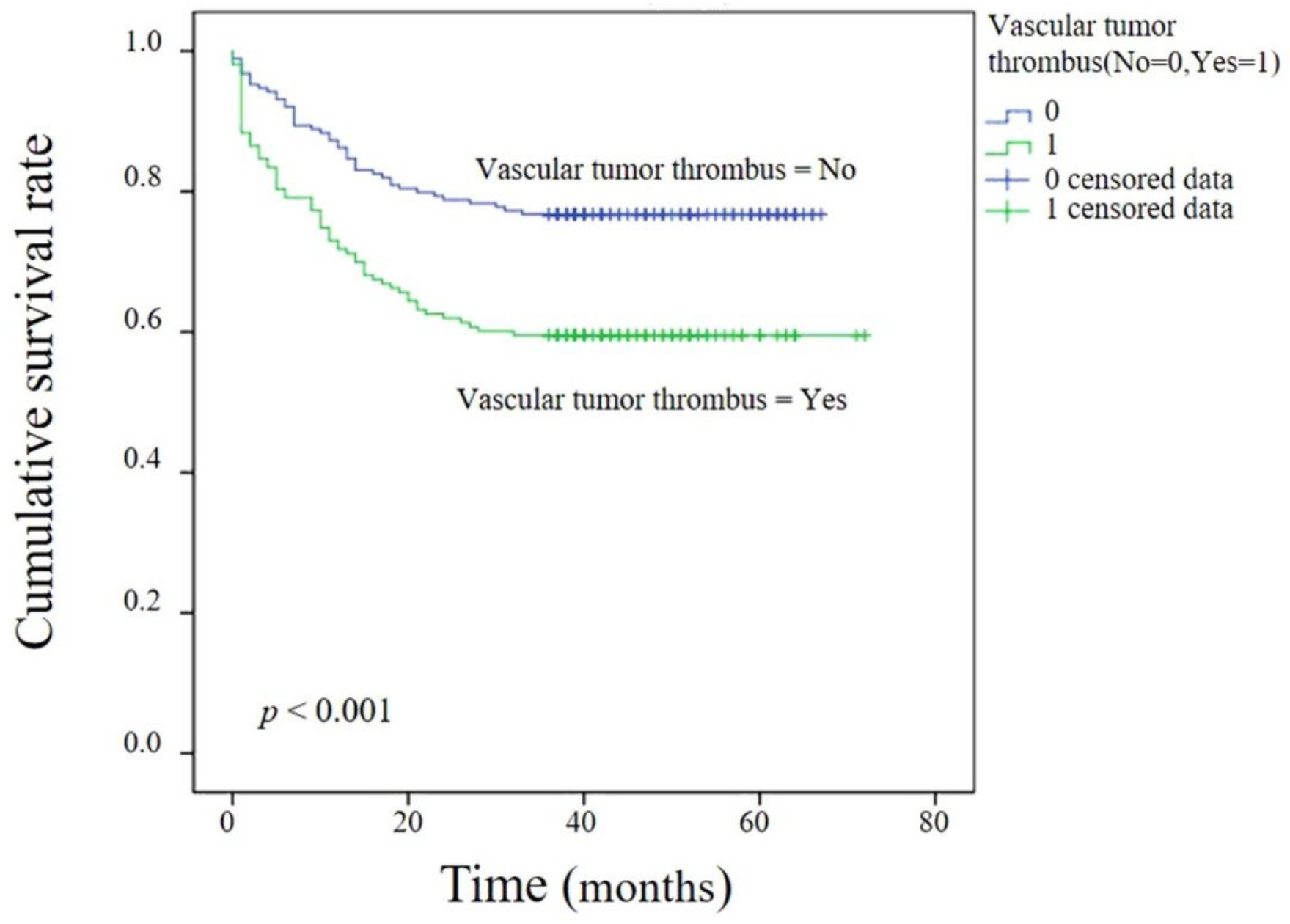

Figure 3

The Kaplan-Meier survival curves of DFS for patients stratified according to prognostic factors: Vascular tumor thrombus 


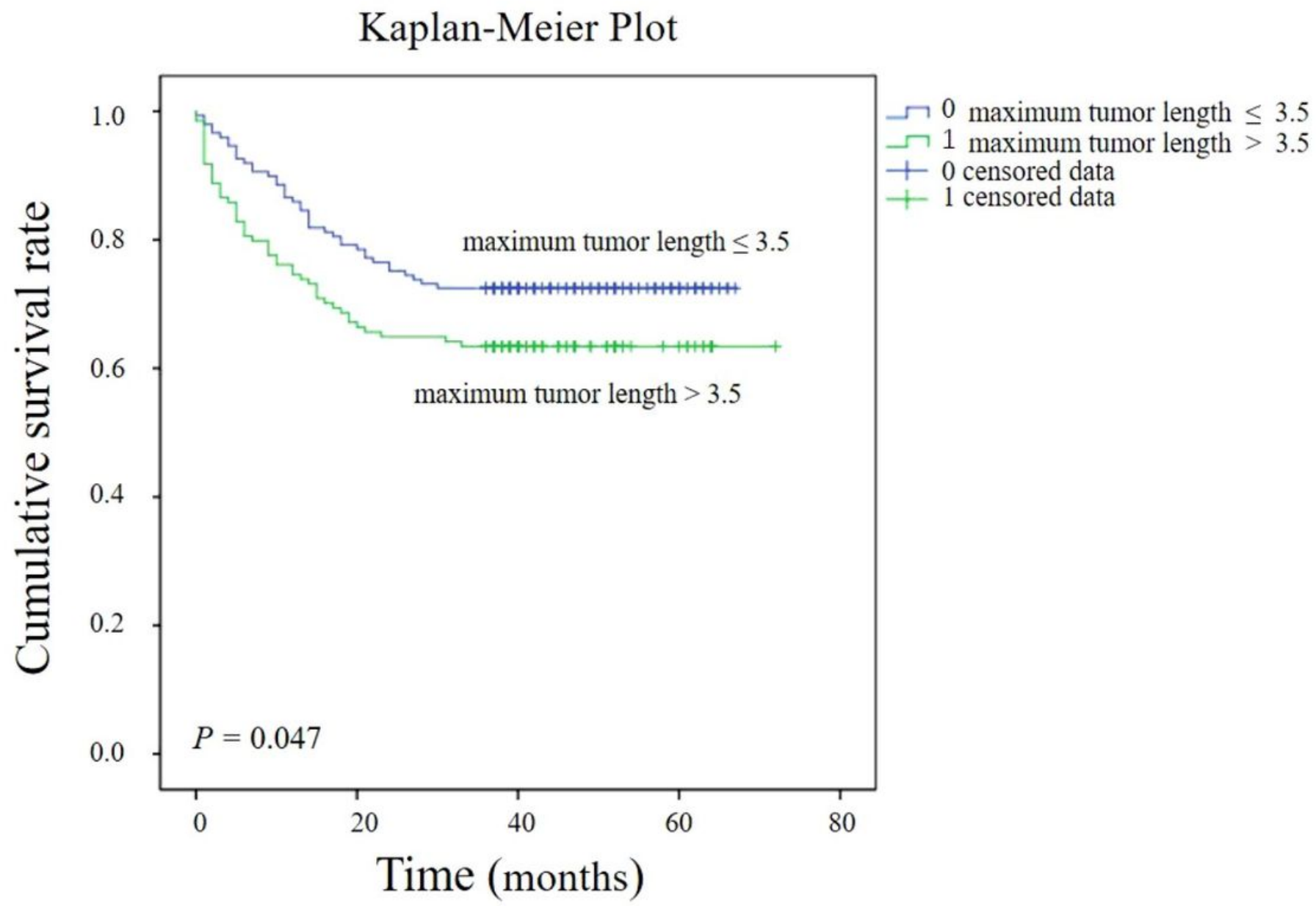

Figure 4

The Kaplan-Meier survival curves of DFS for patients stratified according to prognostic factors: Maximum tumor length 
Kaplan-Meier Plot

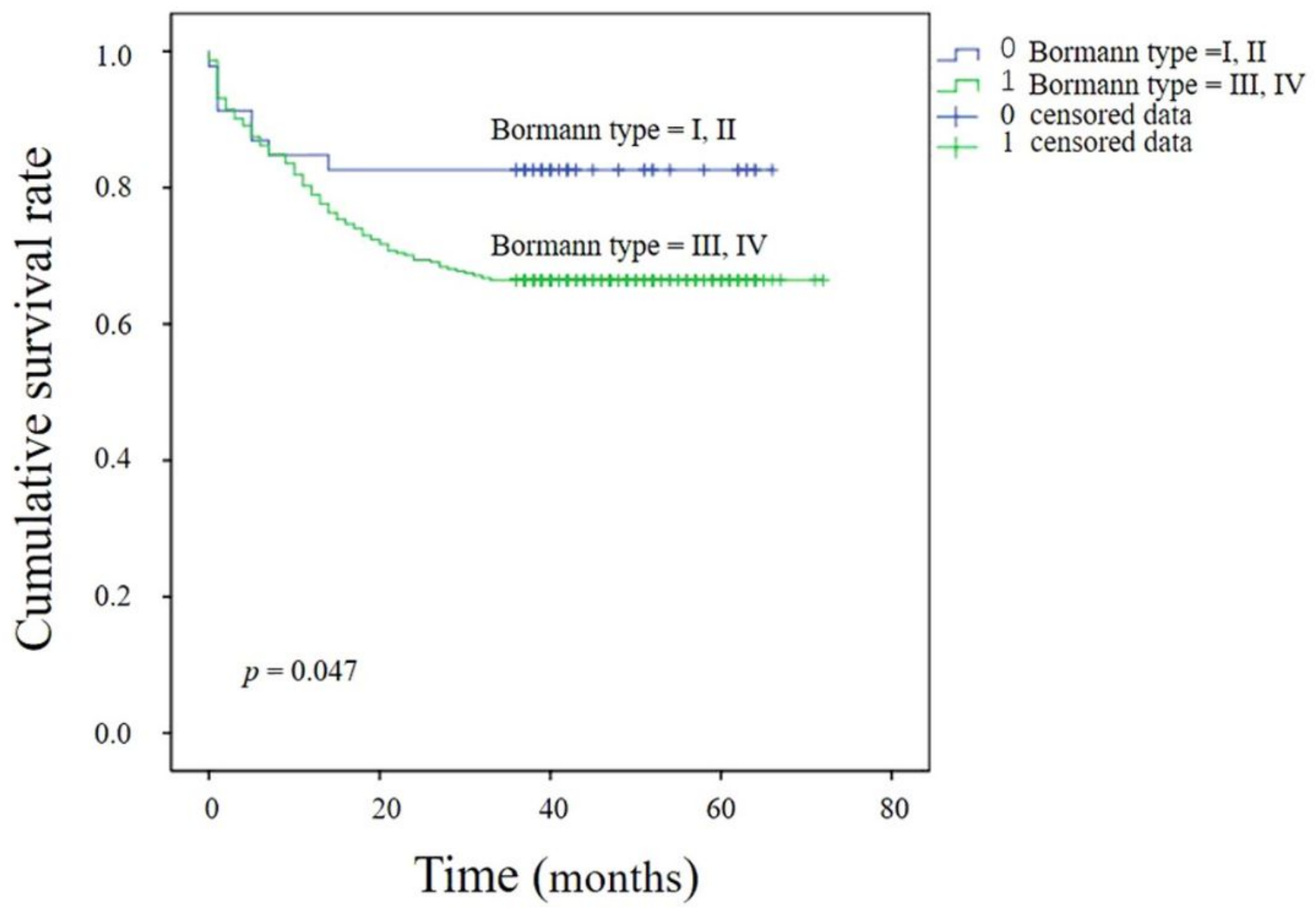

Figure 5

The Kaplan-Meier survival curves of DFS for patients stratified according to prognostic factors: Bormann type 


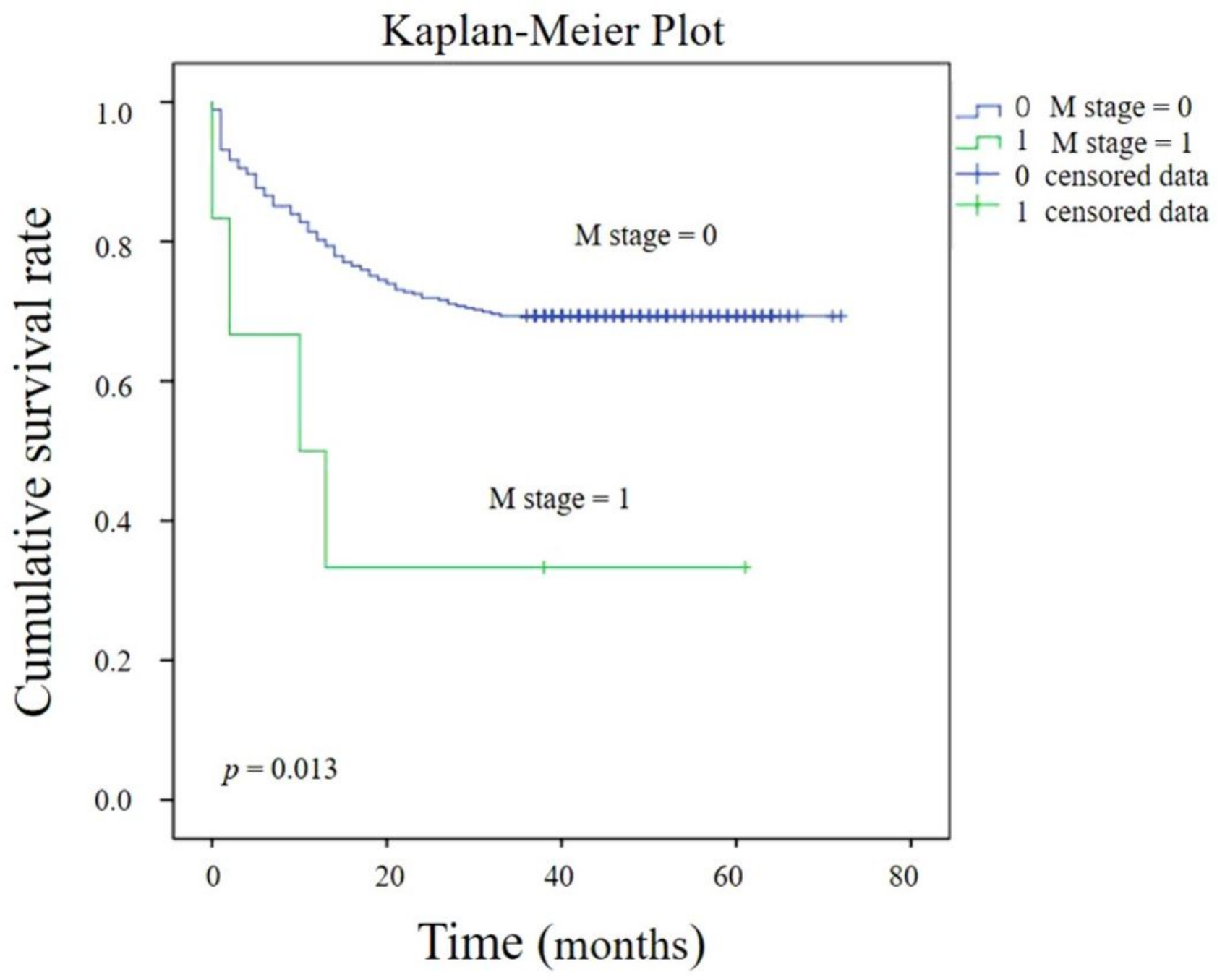

Figure 6

The Kaplan-Meier survival curves of DFS for patients stratified according to prognostic factors: M stage 


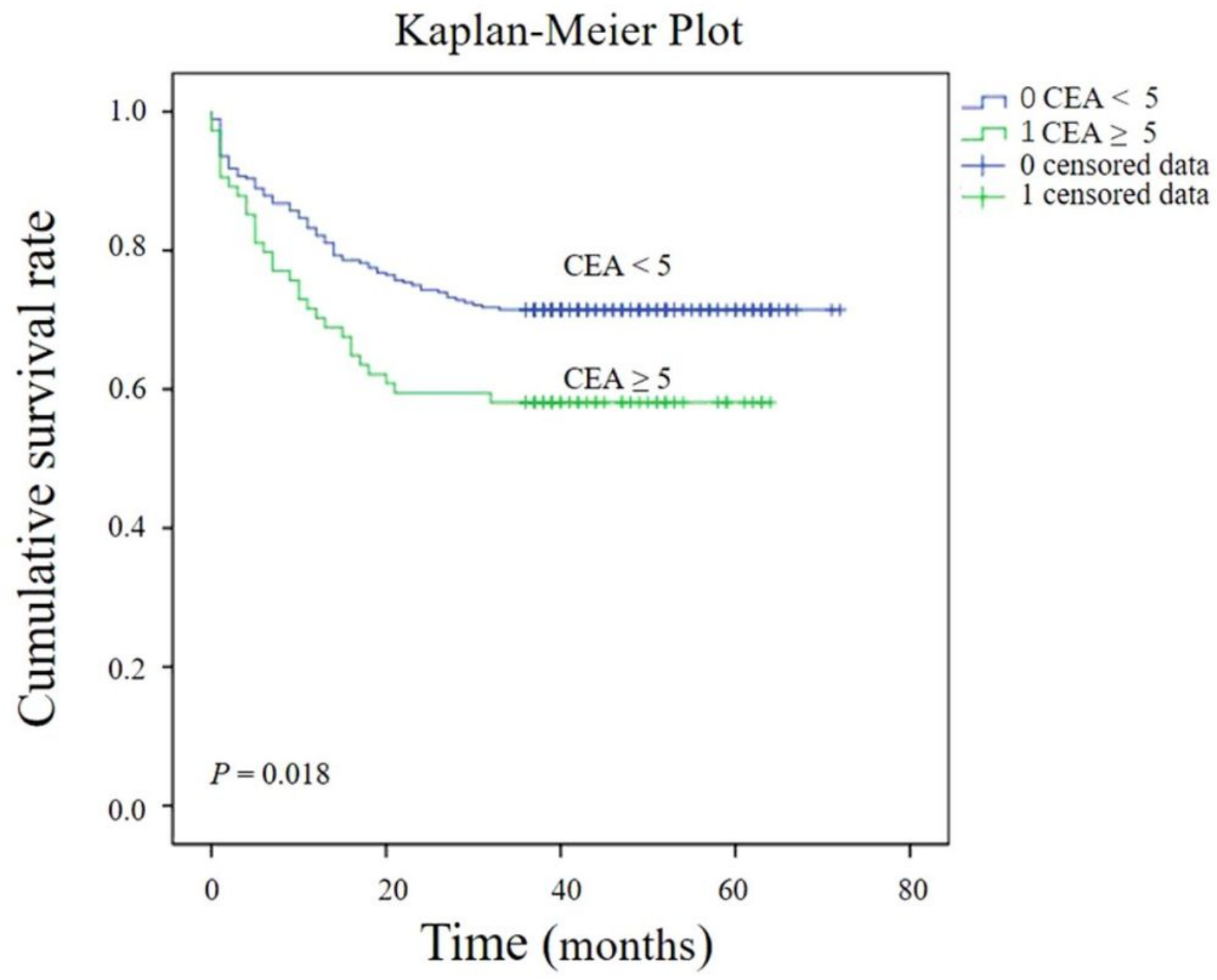

Figure 7

The Kaplan-Meier survival curves of DFS for patients stratified according to prognostic factor: MWR 


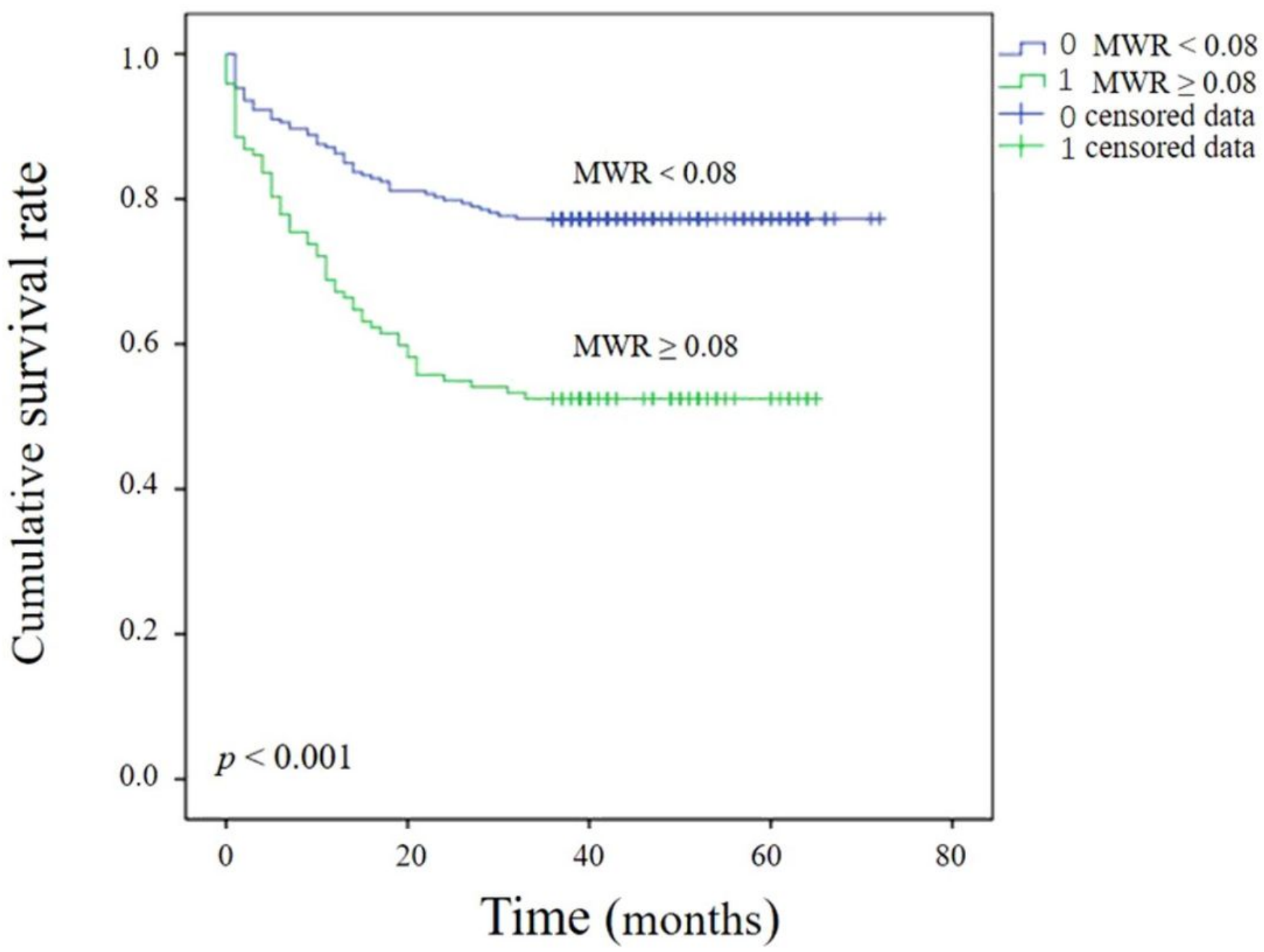

Figure 8

The Kaplan-Meier survival curves of DFS for patients stratified according to prognostic factor: CEA

\section{Supplementary Files}

This is a list of supplementary files associated with this preprint. Click to download.

- Table10.xlsx

- Table9.xlsx

- Table8.xlsx

- Table7.xlsx

- Table6.xlsx

- Table5.xlsx

- Table4.xlsx

- Table3.xlsx 
- Table2.xIsx

- Table1.xIsx 